\title{
ABAKA: a Novel Attribute-Based k-Anonymous Collaborative Solution for LBSs
}

\author{
Tooska Dargahi ${ }^{\mathrm{a}}$, Moreno Ambrosin ${ }^{\mathrm{b}}$, Mauro Conti $^{\mathrm{b}}, \mathrm{N}$. Asokan ${ }^{\mathrm{c}}$ \\ ${ }^{a}$ Department of Computer Engineering, West Tehran Branch, Islamic Azad University, \\ Tehran, Iran \\ ${ }^{b}$ Department of Mathematics, University of Padua, Padua, Italy. \\ ${ }^{c}$ Department of Computer Science, Aalto University and University of Helsinki, Finland.
}

\begin{abstract}
The increasing use of mobile devices, along with advances in telecommunication systems, increased the popularity of Location-Based Services (LBSs). In LBSs, users share their exact location with a potentially untrusted Location-Based Service Provider (LBSP). In such a scenario, user privacy becomes a major concern: the knowledge about user location may lead to her identification as well as a continuous tracing of her position. Researchers proposed several approaches to preserve users' location privacy. They also showed that hiding the location of an LBS user is not enough to guarantee her privacy, i.e., user's profile attributes or background knowledge of an attacker may reveal the user's identity. In this paper we propose ABAKA, a novel collaborative approach that provides identity privacy for LBS users considering users' profile attributes. In particular, our solution guarantees $p$-sensitive $k$-anonymity for the user that sends an LBS request to the LBSP. ABAKA computes a cloaked area by collaborative multihop forwarding of the LBS query, and using Ciphertext-Policy Attribute-Based Encryption (CP-ABE). We ran a thorough set of experiments to evaluate our solution: the results confirm the feasibility and efficiency of our proposal.
\end{abstract}

Keywords: Location-Based Services, Privacy, $k$-anonymity, $p$-sensitivity, Ciphertext-Policy Attribute-Based Encryption.

Preprint submitted to Elsevier

November 3, 2016 


\section{Introduction}

With the rapid development of mobile devices and advances of telecommunications, mobile users tend to have ubiquitous access to information such as traffic prediction or location map data. Location-Based Services (LBSs) are the best examples of this new trend, allowing mobile users to receive information based on their geographical position [1]. Based on their location, mobile users can access several types of information and services, e.g., getting the position of the nearest gas station, restaurant or hospital.

An LBS consists of two major entities: a user (from now on referred also as issuer of a query) who is interested in acquiring location-based service, and a Location-Based Service Provider (LBSP) which provides the desired location-based service to the issuer. To obtain such a service, the issuer sends her geographical location, along with her identity and the query to the LBSP. Unfortunately, some queries (such as searching for the nearest hospital specialized in a particular disease) may reveal privacy-sensitive information about the issuer.

The growing interest of smartphone users in using LBSs leads to two major privacy concerns: location privacy and identity privacy (also known as query privacy). The former refers to preventing the disclosure of the exact location of an issuer, while the latter is the ability of concealing the link between her identity and her query. These two concepts are complementary, and therefore, guaranteeing both location and identity privacy for an issuer becomes a challenging task. Researchers proposed several solutions providing location and identity privacy in the context of LBSs (examples can be found in [2]). The location privacy problem has also been studied extensively in other contexts such as sensor networks [3], 25 and cloud computing [4.

A popular tool used in the literature to guarantee user's identity privacy, in the context of LBSs, is the concept of $k$-anonymity [5]. This concept refers to a set of $k$ users in which a target user is indistinguishable (with respect to her location) from the other $k-1$ individuals in the set. However, according 
profile attributes, we can only guarantee $k$-anonymity by considering anonymity sets in which all the users have the same profile attributes. Furthermore, the authors in 7 . proved that $k$-anonymity is not sufficient to protect the privacy of an individual's attributes in a dataset, and might not prevent the disclosure of sensitive attributes for the user. With respect to sensitive attributes, we refer to a precise definition in [8]: "an attribute whose values may be confidential for an individual (subject to her/his preferences)". Indeed, in the context of LBSs, the semantics of an issued query might allow the LBSP to infer sensitive attributes of an issuer's profile, or even her identity [9].

In order to address this problem, researchers proposed a solution called $p$-sensitive $k$-anonymity [7, 9, 10, in which at least $p$ different values for each group of sensitive attributes are used. In the context of LBSs, this translates in ensuring that the anonymity set for an issuer contains individuals with diverse values for a specific set of privacy-sensitive attributes. In this paper, inspired by

45 the concept of "personalized privacy preservation" by Xiao and Tao in [8], we give the opportunity to the issuer of a query to decide her preferences in sensitive attributes, based on her query content and physical location. We provided this feature for the issuer, due to the fact that an attribute could be sensitive for a query in special location, and insensitive for another query in another location50 (we will further clarify this matter in the following). Before introducing the key contribution of the paper, we present a running example.

Medical help example. Consider a set of smartphone users in a geographical area. We assume that each user is assigned a profile that consists of five attributes: $\{$ Gender, Age, Nationality, Job, Zip-code $\}$. Suppose a user $u_{1}$ is a 19-year-old 55 Finnish girl living in Italy. She is looking for a pregnancy help center near her house, where the doctors are able to speak English. She sends an LBS query $Q=$ "where is the nearest pregnancy help center with English speaking doctors?" and wants to cloak her location while being 9-anonymous. In this example, based on the content of the query, the attributes Gender and Zip-code should be identical between all the users in the anonymity set (i.e., providing 
profile $k$-anonymity). Moreover, based on the semantics of the issued query, Age and Nationality are sensitive attributes of $u_{1}$. It should be noted that age and nationality are not sensitive attributes per se, but due to the fact that the issuer is in Italy, her nationality could reveal her identity. Moreover, her 65 query semantics (i.e., being pregnant) strongly relates to her age. Therefore, we consider these two attributes to be her sensitive attributes. Assume that she computes a cloaked area using one of the existing $k$-anonymity preserving methods, and sends her query to the LBSP. Given the fact that she is looking for an English speaking doctor, a malicious LBSP can infer that the issuer is foreigner. Moreover, suppose that there are only two foreign users in her cloaked area: one 19 years old $\left(u_{1}\right)$ and the other 50 years old. In such case, if the attacker has this background knowledge, he can infer that the issuer is likely to be $u_{1}$. This example emphasizes the fact that, based on the query semantics and considering the attacker's background knowledge, some attributes could be sensitive in specific scenarios and reveal the identity of the issuer. A proper privacy preserving solution should take into account sensitive attributes of $u_{1}$, according to the semantics of the query. For example, a solution could provide an anonymity set in which all the $k$ users are non-Italian (i.e., providing profile $k$-anonymity) and there are enough diversity in age attribute (i.e., providing so $p$-sensitivity considering the more probable values for being pregnant).

Contribution. In this paper, we propose ABAKA (Attribute-Based $k$-Anonymous collaborative solution for LBSs), a novel solution to provide both identity, and location privacy for LBS users taking into account the profile attributes of the users. Our motivation is the existing limitations of the prior research in the area of LBS users' privacy: on the one hand, those researches which attempt to ensure k-anonymity considering the profile of the users (such as in [6]) are centralized; and on the other hand, the existing distributed approaches do not consider profile attributes of the LBS users (such as in 11]).

In this paper, we make the following contributions:

- We propose ABAKA, the first privacy-preserving LBS system that guar- 
antees p-sensitive k-anonymity running a TTP-free protocol between participating users (Section 4). In particular, ABAKA has the following features:

- It cloaks the exact location of a user into a cloaked area of arbitrary size, by ensuring that (at least) $k-1$ collaborating users will forward a query in a random multi-hop path within the cloaked area.

- ABAKA guarantees $p$-sensitivity by ensuring that the collaborating users in the anonymity set, which will forward the query, have specific attributes selected by the issuer. Each issuer can select a desired set of attributes based on the semantics of the query she wants to send. In particular, with ABAKA she can decide: (i) which attributes need to be identical within an anonymity set; and (ii) which attributes are sensitive, and thus need to have $p$ different values within the anonymity set.

- ABAKA adopts Ciphertext-Policy Attribute-Based Encryption (CPABE) [12, in order to apply fine-grained access control over encrypted data, by defining high-level access policies as a combination of attributes. $\mathrm{CP}-\mathrm{ABE}$ allows the issuer to specify attribute-based policies on the query; in this way, she ensures that other $k-1$ collaborative users have the desired attributes.

- ABAKA ensures the confidentiality of the query, by using public key encryption.

- We run a systematic performance evaluation of ABAKA using two different datasets (Section 5.1) and a thorough evaluation of the computational overhead imposed by cryptographic processing required by ABAKA (Section 5.2. . Our evaluation demonstrates that ABAKA is feasible on both smartphone and PC platforms. 


\section{Background on Attribute-Based Encryption}

In what follows, we introduce the fundamental concepts about Attribute-

120

CP-ABE) in particular. In 2005, Sahai and Waters introduced a Fuzzy IdentityBased Encryption scheme [13, called ABE. This scheme is a public key encryption protocol that allows an encryptor to specify fine-grained access control policies over data. In this scheme, each user is assigned a set of attributes (e.g., Gender, users that have a specific set of attributes will be able to decrypt the ciphertext (i.e., if user's attributes satisfy the policy over the data). CP-ABE [12] is a type of $\mathrm{ABE}$ in which the access policy is included into the ciphertext, and expressed as a combination of attributes. An example of such a policy is: $($ Age $=19 \wedge$ Gender $=$ female $) \vee($ Nationality $=$ Italian $)($ see Figure ?? $)$.

Each user has a private decryption key, which represents the set of attributes she owns. She will be able to decrypt a ciphertext if and only if a subset of her attributes satisfies the access policy on the data. By construction, in the CP-ABE scheme only the key issuer (i.e., a Certificate Authority) is able to generate new private keys, therefore preventing collusion attacks 12 .

In general, a CP-ABE scheme provides the following functions:

- Setup. It takes as input an implicit security parameter, and outputs the public key $p k$, and the master key $M K$.

- Encryption. It takes as input a message $M$, an access policy $A$, and the public key $p k$, and outputs the corresponding ciphertext $E$.

- KeyGen. It takes as input a set of attributes $\mathcal{A}=\left\{A_{1}, A_{2}, \cdots, A_{n}\right\}$, the master key $M K$ and the public key $p k$. It outputs a decryption key $D$ reflecting the given attributes.

- Decryption. It takes as input the ciphertext $E$ that is encrypted under 
and the public key $p k$. It outputs the message $M$ if and only if $\mathcal{A}$ "satisfies" the access policy $P$.

Several researches adopt ABE to provide access control and location privacy. For example, adopting CP-ABE, Dargahi et al. [14 proposed a $k$-anonymous

\subsection{System Model}

We consider a set of users $U=\left\{u_{1}, u_{2}, \cdots, u_{m}\right\}$ in a geographical area. Each user can be a potential LBS user (i.e., an issuer) and is equipped with a location-aware wireless device (e.g., smartphone or tablet) that is able to retrieve stationary (from the time the issuer sends out the query until when she receives the response back), or to have limited mobility. Users can communicate with their neighboring users over a wireless medium (e.g., via WiFi) via a single-hop 
or a multi-hop route. Moreover, we assume that users ignore received packets

Table 1: Notation table.

\begin{tabular}{|c|c|}
\hline Notation & Description \\
\hline$Q, R$ & Location-based query and response, respectively \\
\hline$s, r$ & Issuer-generated random numbers \\
\hline$p k_{L}, s k_{L}$ & $\begin{array}{l}\text { Respectively, public and private key pair of the } \\
\text { LBSP }\end{array}$ \\
\hline$k_{u}, s k_{u}$ & $\begin{array}{l}\text { Respectively, symmetric key and private CP-ABE } \\
\text { key of } u\end{array}$ \\
\hline$k_{r}$ & Symmetric key of collaborating users \\
\hline$p k$ & Public CP-ABE key \\
\hline $\mathrm{CPABEENC}_{p k}(p t x t, p)$ & $\begin{array}{l}\text { Encryption of a plaintext } p t x t \text { applying a policy } \\
p, \text { with } \mathrm{CP}-\mathrm{ABE}\end{array}$ \\
\hline $\mathrm{ENC}_{k}(p t x t)$ & $\begin{array}{l}\text { Symmetric encryption of a plaintext ptxt, using } \\
\text { key } k\end{array}$ \\
\hline
\end{tabular}

We assume that each user is assigned a profile which consists of a set of attributes $\mathcal{A}=\left\{A_{1}, A_{2}, \cdots, A_{n}\right\}$. These attributes can be of different types: personal information (e.g., gender), employment information (e.g., job), and contact information (e.g, Zip-code). In our medical help example, we consider the following profile attributes: $\left\{A_{1}:\right.$ Gender, $A_{2}:$ Age, $A_{3}:$ Nationality, $A_{4}$ : $J o b, A_{5}:$ Zip-code $\}$. We also assume that none of the users have exact information about the number of users in her vicinity, and their profile attributes. We consider the LBSP to be untrusted, and assume that each LBS user does not want to share her exact location and identity $(I D)$ with the LBSP. In our 
model, the issuer sends her request to the LBSP through a multi-hop path, to anonymize her location and identity. Our multi-hop approach is similar to the work in [20, 22, however in ABAKA the issuer looks for a set of collaborating users having specific attributes, who cooperate with each other to anonymize the location of the issuer. We also assume that each user, based on its own policy, decides whether to participate in the anonymizing process. One may think of an incentive mechanism in order to motivate users to participate in our collaborative scheme. There are several monetary and non-monetary incentive schemes in the literature 23, which could be considered to be a complement for ABAKA. One possible approach, to be used, could be the privacy-aware incentive mechanism proposed in 24, which is a TTP-free scheme based on blind signature. However, an encouraging mechanism is out of the scope of this paper (and an orthogonal open research problem, as pointed out by Conti et al. 25]), and we leave it as future work.

We assume that the LBSP has a pair of keys: a public key $p k_{L}$, and a private key $s k_{L}$ that are used to preserve confidentiality and integrity of the message sent by the issuer to the LBSP. Moreover, we suppose that there could be multiple Certification Authorities (CAs) [26], each of which being responsible for a specific geographical area (e.g., states or municipalities), to authenticate the users and assign them CP-ABE private keys (users key management is out of the scope of this paper). Each user obtains a CP-ABE private key based on her profile attributes, from the CA nearest to her location. The CP-ABE private key will be used for authentication of collaborating users, and fulfilling the requirement of $p$-sensitivity. Furthermore, CAs provide the CP-ABE public key, that the issuer uses to encrypt her query specifying an access policy. In our solution, we assume each user to contact the nearest CA when her profile attributes change, in order to retrieve a new $\mathrm{CP}-\mathrm{ABE}$ private key. Note that this does not change the collaborative nature of our approach. We also assume each user $u_{i}$ has a symmetric key, $k_{u_{i}}$, which can be a random number defined by $u_{i}$. The user $u_{i}$ will use this key to encrypt/decrypt a special field of the packet during the packet forwarding procedure. Moreover, the issuer generates a random group 
secret key, $k_{r}$, for the collaborating users.

Finally, in our model each user can specify her privacy requirements in terms of size $k$ of the anonymity set, number of users with specific issuer-defined attributes $p$, and the largest and smallest desired cloaked area size. Also, we assume the issuer to not issue any query that the query content could lead to her identification or reveal information about her exact location (otherwise the use of anonymity preserving approaches would not make much sense).

\subsection{Adversary Model}

We consider two types of adversaries: passive and active. A passive adversary can be one of the following three entities [27, 11]: (i) the untrusted LBSP, which collects information about LBS users such as their location, identity or activities, based on their queries; (ii) an outsider eavesdropper on wireless communication, which is interested in identifying location and identity of the issuer; (iii) the users that collaborate in computing the $k$-anonymity set. The collaborating users are not fully trusted; we consider them to be honest-but-curious (we observed that this assumption is consistent with several works in the literature, such as the ones in $[28,29,30])$ : i.e., users honestly follow the ABAKA protocol, and neither drop nor modify the packets. However, they are curious to learn location and identity of the issuer, or of the other users in the $k$-anonymity set. We assume that a malicious user cannot generate fake profiles in order to participate in our protocol and decrease the privacy level of the issuer, since the CAs authenticate the users upon joining the network and assign them CP-ABE private keys (we found this assumption consistent with [31, 32]).

An active adversary can be one of the non-collaborating users who is not able to satisfy the access policy on the encrypted packet (i.e., the user who does not have the issuer-defined attributes). He is interested in identifying the issuer, modifying the LBS request, or reducing the issuer's privacy level. In the last case, he aims at reducing the number of users in the cloaked area (i.e., reducing the value of $k$ ). We assume that both passive and active adversaries have some background knowledge about the users [27. This background information could 
be about profile attributes of the users, such as location information (e.g., office address), personal information (e.g., age or nationality), or even the exact or estimated number of users in a geographical location. The adversary aims at using his background knowledge to attack the privacy of the issuer. In our model, we address the collusion attack of non-collaborating users and we assume that collaborating users do not collude (as they are semi-trusted). Finally, in this paper we do not consider other types of attacks, such as, Denial of Service, which is inevitable in all the collaborative approaches in wireless networks.

\section{Our solution: ABAKA}

In this section, we present ABAKA, our TTP-free solution that provides identity privacy for LBS users. ABAKA deals with both generating and sending the LBS query to the LBSP (Section 4.1), as well as generating and forwarding the requested location-based service to the issuer.

First, the issuer $u_{i}$ divides the encrypted query into $k-1$ parts, and on each part enforces a specific access policy by means of CP-ABE [12]. Then, the issuer sends the packet to the LBSP through a multi-hop path. This way, she conceals her identity among other $k-1$ neighboring users who are able to decrypt the CP-AB encrypted parts of the packet. Figure 1 provides a high-level example of our multi-hop attribute-based solution, considering $k=3$. As Figure 1 shows, the protocol cloaks the position of the issuer (by collaboration of both users with green tick icon and red cross icon in Figure 1 and computes a $k$-anonymity set based on the issuer-defined attributes. Using CP-ABE allows us to address two important issues:

275

- Finding $k-1$ collaborating users (users with green tick icon in Figure 1) having specific attributes, which could be issuer's sensitive attributes. Enforcing a policy on each of the $k-1$ parts of the message, the issuer will be sure that only the users with attributes satisfying the policy, are able to decrypt one part. Thus, we guarantee that the collaborating users in 

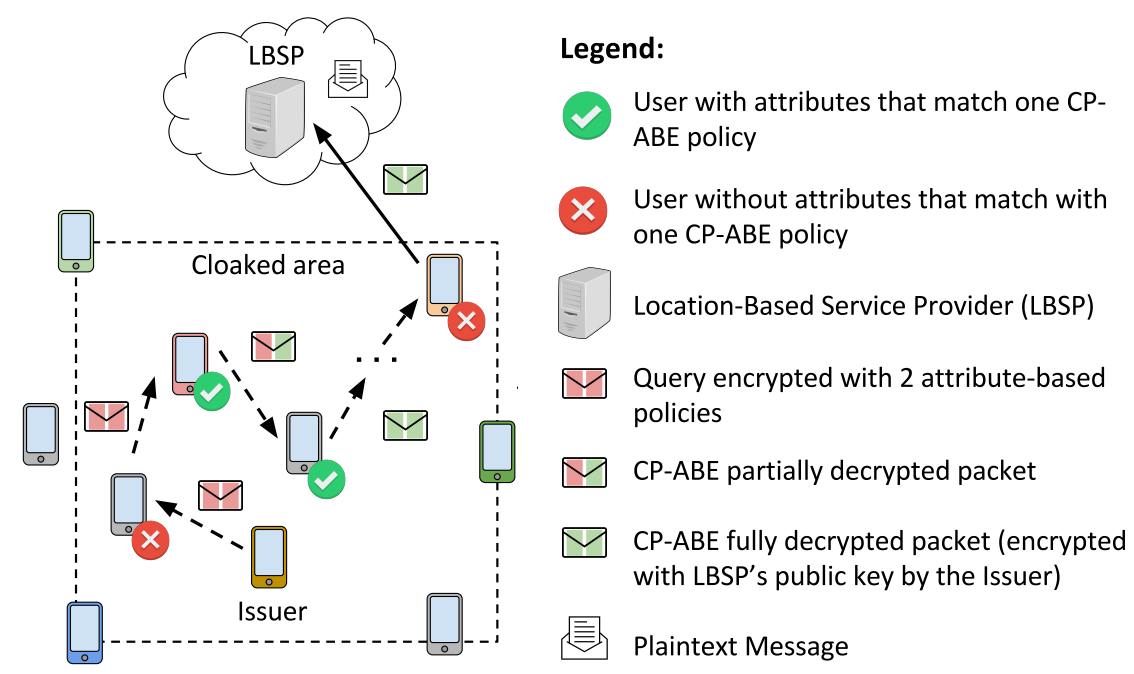

Plaintext Message

Figure 1: Multi-hop CP-ABE based routing to form a rectangle cloaked area, example with $k=3$.

the $k$-anonymity set satisfy $p$-sensitivity (recall that collaborating users are honest-but-curious). We assume that each collaborating user uses her CP-ABE private key only one time for each received packet. In other words, we assume that if she is able to decrypt some of the CP-AB encrypted parts of the packet with her private key (satisfying more than one policy), she will process just one part. We consider this assumption to ensure that all the $k-1$ parts of the message will be processed by $k-1$ different collaborating users and hence ensuring the $k$-anonymity.

- Addressing privacy attack form non-collaborating users, i.e., users outside the cloaked area in Figure 1. As non-collaborating users are not able to satisfy any of the access policies, they will not be able to decrypt any of the query parts. Therefore, they will not be able to reduce the privacy level of the issuer by collaboration in computing the cloaked area.

In our medical help example, user $u_{1}$ wants to be 9 -anonymous between eight other users who are female and have the same four digit prefix Zip-code, i.e., Gender $=$ female and Zip-code $=0019$. Moreover, due to her sensitive attributes, she is looking for eight other users who are not Italian and have 
diverse values for the age attribute which fall in three different age categories, i.e., $15 \sim 24,25 \sim 34$, and $35 \sim 44$. User $u_{1}$ uses ABAKA to conceal her identity.

Th this section, we describe how a query issuer, $u_{i}$, is generating and forwarding an LBS request to the LBSP. In particular, an LBS request packet is composed 
of the six fields illustrated in Figure 2 and discussed in the following.

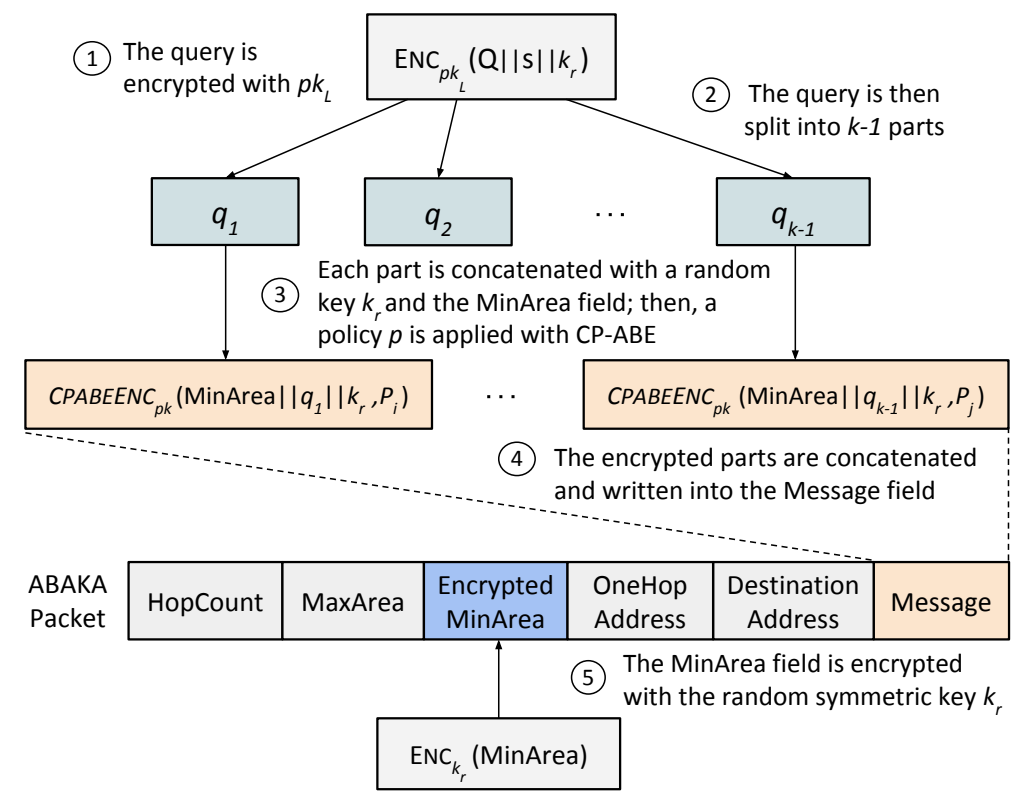

Figure 2: LBS request packet format generated by the issuer.

The Message field contains the query $Q$, a random number $s$, and a randomly generated symmetric key $k_{r}$ encrypted with the public key, $p k_{L}$, of the LBSP. This message is then split into $k-1$ parts, each encrypted with CP-ABE applying a certain policy, and finally recomposed. The HopCount field denotes the maximum number of hops that the packet should pass through other users. Its value should be greater than $k-1$. The MaxArea field denotes the maximum size of the desired cloaked area in the form of a rectangle, which is defined by two points $\left(x_{l}, y_{l}\right)$ and $\left(x_{r}, y_{r}\right)$ for bottom left and top right corners of the rectangle, respectively. The MinArea field represents the minimum size of the desired cloaked area in the form of a rectangle, which is defined by two points $\left(x_{l}^{\prime}, y_{l}^{\prime}\right)$ and $\left(x_{r}^{\prime}, y_{r}^{\prime}\right)$ for bottom left and top right corners of the rectangle, respectively. The content of this field is encrypted with the randomly generated symmetric key $k_{r}$. After completing the cloaking procedure, this field represents the actual cloaked area dimensions. OneHopAddress is used for routing back the LBSP response to the issuer of the query. The initial value of this field is $\operatorname{ENC}_{k_{u_{i}}}(r)$, 
where $r$ is a random number generated by the issuer $u_{i}$. Upon receiving the LBS request packet, each user encrypts the address of the previous hop with her symmetric secret key $\left(k_{u_{i}}\right)$ and appends this encrypted layer to the current content of the OneHopAddress field. Finally, DestinationAddress contains the address of the LBSP.

\subsubsection{Packet Generation}

An issuer $u_{i}$ generates a packet executing the Algorithm 1] which comprises the following steps:

Step 1. The query issuer, $u_{i}$, generates a Message which comprises her query, $Q$, a random number, $s$, and a randomly generated symmetric key $k_{r}$ encrypted with the public key, $p k_{L}$, of the LBSP (Algorithm 1, lines 2-3).

Step 2. The issuer splits the encrypted Message into $k-1$ parts (e.g., in chunks of equal size), where $k$ is the $k$-anonymity parameter (Algorithm 1 , line 4 ). Then, she defines the minimum size of the desired cloaked area, MinArea field (Algorithm 1, line 5). She appends the MinArea field and also the symmetric key $k_{r}$ to each part and encrypts that part with CP-ABE, specifying an access policy, i.e., a combination of desired attributes (Algorithm 1 , lines 6-8). The reason behind including MinArea field in each part is to provide each collaborating user with the means of checking whether the actual minimum desired cloaked area defined by the issuer has been modified during the path by intermediate nodes (we will provide a further discussion in Section 4.2).

Step 3. The issuer creates an empty packet (Algorithm 1, line 9), as illustrated in Figure 2. Then, she concatenates the $k-1$ parts generated in the previous step to form a complete message (Algorithm 1, line 10). Afterward, $u_{i}$ defines her privacy requirements in terms of maximum number of neighbors that the message should pass through, the maximum and minimum size of the desired cloaked area, and the destination address, i.e., the address of the LBSP (Algorithm 1, lines 11-14). The issuer $u_{i}$ encrypts the MinArea field of the header with $k_{r}$, to avoid eavesdroppers or non-collaborating users to be able to read (or modify) such information (Algorithm 1, line 13). 


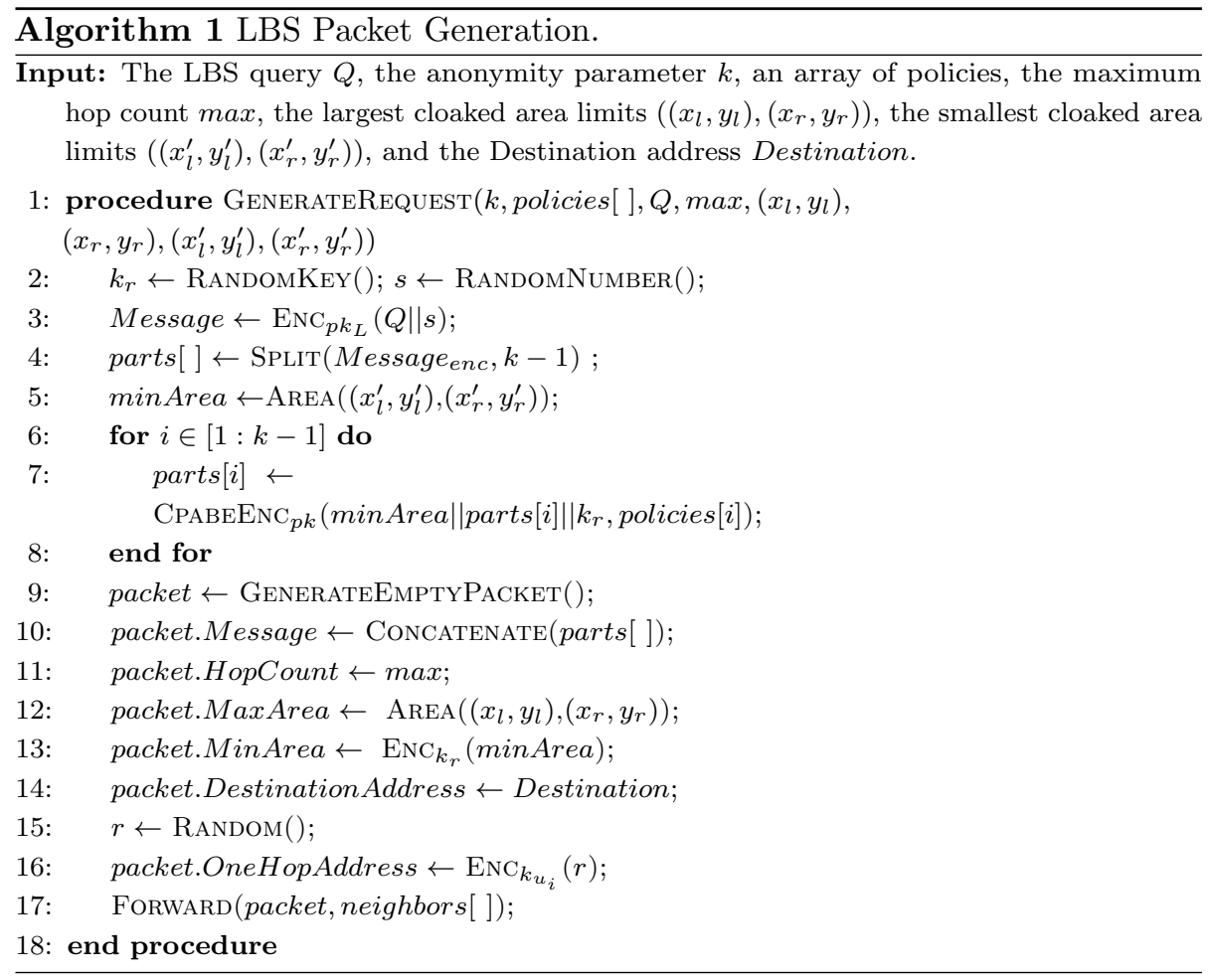

Step 4. Before sending the packet to a next hop, $u_{i}$ encrypts a random number $r$ with her symmetric secret key $\left(k_{u_{i}}\right)$, and attaches it to the packet (Algorithm 1, lines 15-16). Finally, $u_{i}$ sends the generated packet to one of her neighbors. The choice of the next-hop can be done in several ways, e.g., selecting randomly or based on the proximity with the issuer (Algorithm 1, line 17).

In the medical help example, user $u_{1}$ splits the encrypted query into eight parts. Then, she defines her desired smallest cloaked area (MinArea) which could be $100 m \times 100 m$ rectangle including her house (the house is not necessarily placed in the center of the defined area). She concatenates the MinArea to each part along with a random symmetric key $k_{r}$, and applies the aforementioned policies on each part. Afterward, she determines her largest desired cloaked area, MaxArea, which is a $600 m \times 600 m$ rectangle including her geographical position and the maximum number of hops (e.g., HopCount=15). Then she encrypts a random number $r$ with her symmetric key $\left(k_{u_{1}}\right)$ and specifies the address of the 
LBSP. Finally, she forwards the generated packet to one of her neighbors.

\subsubsection{Packet Forwarding}

Once received a packet, a user $u_{j}$ performs the following operations (the packet forwarding procedure's flowchart is depicted in Figure 3):

Step 1. User $u_{j}$ checks whether she resides in the largest desired cloaked area defined in the MaxArea field of the packet.

Step 2. If $u_{j}$ resides in the defined area, she peruses the packet fields

395

to decide, based on her own policies, whether she wants to participate in the cloaking algorithm. If she does not want to collaborate, she forwards the packet to another user. Otherwise, she performs the following actions:

- Step 2.1: The user $u_{j}$ checks the Message field of the packet, to verify whether there is any encrypted part, and if she is able to decrypt one of them. User $u_{j}$ will be able to decrypt one part, if and only if the attributes associated to her profile (i.e, attributes associated to her private key $s k_{u_{j}}$ ) satisfy the policy enforced on that part. If able to decrypt, $u_{j}$ decrypts the MinArea field of the packet header, i.e., Packet.MinArea, using the key $k_{r}$ obtained from the CP-ABE decrypted part. Then, $u_{j}$ compares such field with the Part.MinArea field: if Packet.MinArea < Part.MinArea, it means that an attacker has decreased the original value defined by the issuer. In such a case, $u_{j}$ discards the packet. Otherwise, $u_{j}$ continues by checking whether she resides in the area defined by the Packet. MinArea. If not, $u_{j}$ enlarges the area to include also her location. Then, she updates the part she is currently processing, by removing the Part.MinArea field and $k_{r}$ and encrypting such part with $k_{r}$.

- Step 2.2: The user $u_{j}$ updates the current value of the OneHopAddress concatenating the address of the previous hop, and encrypting the whole content of the field with her symmetric secret key $\left(k_{u_{j}}\right)$. This way she adds a new "onion layer" that will be used to route the response message back to the issuer. Then, $u_{j}$ decrements the value of the HopCount field. 


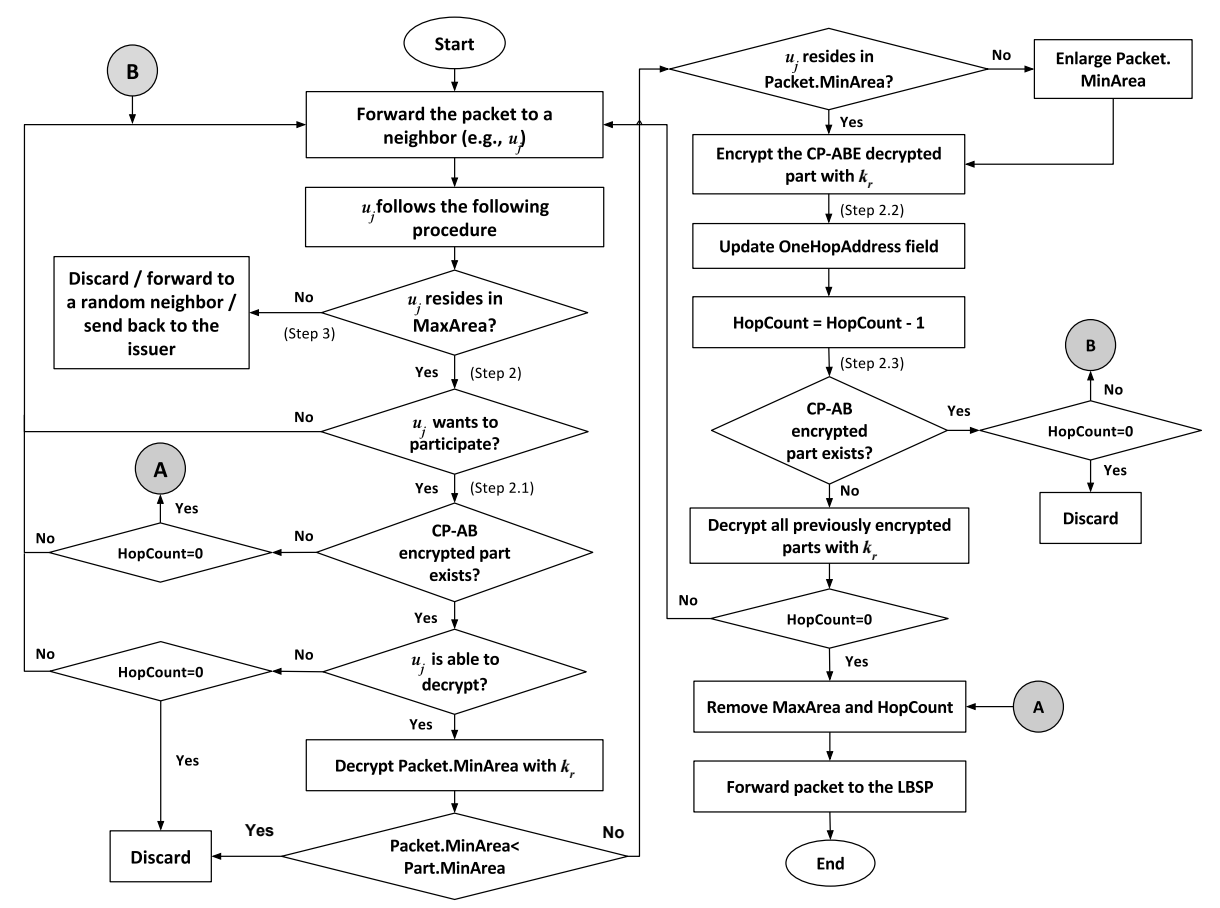

Figure 3: Packet forwarding flowchart.

If $u_{j}$ is the one who decrypted the last part with her CP-ABE key, she decrypts all the previous parts with the key $k_{r}$. Then, if HopCount $=0$, $u_{j}$ removes the MaxArea and HopCount fields of the packet header, and sends the query to the LBSP. The coordinates $\left(x_{l}^{\prime}, y_{l}^{\prime}\right)$ and $\left(x_{r}^{\prime}, y_{r}^{\prime}\right)$ in the Packet.MinArea field represent the actual cloaked area, i.e., the smallest area covering the positions of all the collaborating users. If HopCount $>0$, $u_{j}$ continues forwarding the packet to one of her neighbors.

- Step 2.3: If there are other encrypted parts (i.e., the packet did not pass enough users to guarantee $k$-anonymity), or if the user was not able to decrypt one of the parts of the message, $u_{j}$ continues forwarding the packet to one of her neighbors. Before forwarding the packet, $u_{j}$ checks the HopCount value. If HopCount $=0, u_{j}$ discards the packet. Otherwise, forwards the packet again. 
Step 3. If $u_{j}$ does not reside in the defined largest cloaked area, she can perform one of the following actions: drop the packet, forward it to a random neighbor, or send the packet back to the previous user.

The protocol explained in this section ensures that the query is forwarded through, at least, $k-1$ neighboring users having specific attributes, ensuring $k$-anonymity and $p$-sensitivity.

\subsection{Discussion}

In this section we briefly discuss issues related to packet generation and forwarding, as well as the privacy level provided by ABAKA.

\subsubsection{Packet Generation}

To ensure that the smallest cloaked area specified by the issuer will be respected, we introduced the MinArea field in the ABAKA packet. This field is of extreme importance in order to guarantee the desired privacy level for the query issuer. Indeed, on one hand, an attacker might want to increase such area to reduce the quality of service; and, on the other hand, the attacker might also want to reduce the value of the MinArea field, in this case attempting to reduce the privacy guarantees of the ABAKA. In order to prevent these two attacks, we place the MinArea field inside each of the CP-ABE encrypted parts of the query. We also encrypt the MinArea field of the packet header with a secret symmetric key $\left(k_{r}\right)$, which can be accessed only by the collaborating users after decrypting ${ }_{450}$ a CP-ABE part. This way, only the collaborating users are able to modify this field as well as verifying the possible malicious modifications to the packet, and eventually discarding it. Similarly, also the MaxArea and HopCount fields might be targeted by an attacker, who may want to enlarge or reduce their values. However, such possible attacks would lead to a Denial of Service, that is out of the scope of this work.

\subsubsection{Packet Forwarding}

During the packet forwarding process, we may have some concerns. First, participating in the ABAKA protocol may threaten the privacy of the collaborating 
users. Indeed, the issuer could infer that there are people with specific attributes in computing the cloaked area to support also dynamic networks (e.g., taking into account the speed of the collaborating users, and computing how much they could move by the time the response comes back, and computing whether 

depends on several parameters (e.g., its domain of application), and requires a trade-off between privacy level, overhead, and trust to some central entities (such a trade-off is a common issue in collaborative approaches, such as in [34]). We leave the management of nodes' mobility as a future work.

495 a cloaked area. In this case, the LBSP might identify the user by correlation of the requests over time. In such case, overtime if the other individuals in the anonymity set are changed, then the user $u$ could be the one who is requesting the same query. This attack can happen in two cases: (i) if the attacker has anonymous routing, (ii) if the attacker has local real-time knowledge about the individuals in the set and the query content, and also have historical information about the previous same requests and the individuals in that sets. We leave a thorough study of the latter attack as future work. design choice, we attributed higher priority to users' privacy, with respect to the quality of service. Therefore, in the case of not finding enough collaborating users, the issued query will not be submitted to the LBSP and the issuer will still be anonymous, but we do not ensure that she will receive her requested 520

Finally, another issue is the delay imposed by the multi-hop forwarding, and finding $k-1$ users with specific attributes. ABAKA is most effective in dense environments (in which the probability of finding collaborating users in vicinity is high) and non real-time scenarios. It provides a strong privacy protection considering the issuer profile attributes varying for each user and query, with the cost of imposing delay to the system. In many applications, the issuer is willing to accept a trade-off between strong privacy protection (by defining strict access policies) and latency (or not receiving response at all). We could also define a maximum time bound for the reception of the response: if the issuer does not receive the response within a certain time frame, she can decide to relax the privacy constraints and re-issue the query. It is worth mentioning that, as a service. 


\subsubsection{Privacy Discussion}

As introduced in Section 3.2 , we consider the following adversaries separately: (i) the untrusted LBSP; (ii) an outsider eavesdropper; (iii) the semi-trusted collaborating users; (iv) the untrusted non-collaborating users. We now discuss how ABAKA protects users against these adversaries.

(i) Consider the medical help example. Based on the content of the query, the LBSP could infer that the sender is a foreign woman, probably between 15 and 45 years old. However, even with background knowledge about profile attributes of women in that area, it could not infer which of these women could be the issuer. In fact, there are at least nine women in the age range between 15 and 44, with different nationalities.

(ii) The outsider eavesdropper observes the communication between the users. He is not able to access the content of the packet since it is encrypted with $\mathrm{CP}-\mathrm{ABE}$, and with the public key of the LBSP. If he can observe all the path, 535 he can find out the issuer and if he has background knowledge about what could be the issuer's query, he may only be able to infer some attributes of the collaborating users; however, it is a strong assumption about the adversary. One can think about an on top anonymized routing layer which could be an orthogonal solution to be used along with the ABAKA, and we leave it as a future work.

(iii) There is no useful information inside the LBS packet for honest-butcurious collaborating users; the content of the message is encrypted with the public key of the LBSP, and both location and identity of the issuer are hidden. A curious collaborating user could obtain only knowledge about attributes of all the collaborating users, or, at least, attributes of a subset of collaborating users.

(iv) Non-collaborating users may try to reduce the privacy level of the issuer (e.g., in the previous example, a man could try to collaborate in computing the cloaked area to decrease the value of $k$ ) or to modify the packet. Using CP-ABE, users without specific attributes are not able to decrypt the packet. Therefore, 
reduce the privacy level for the issuer.

\section{Experimental Results}

In this section, we present an experimental evaluation of ABAKA, using two different datasets. In Section 5.1 we provide performance evaluation of ABAKA in terms of success rate considering different scenarios; while in Section 5.2 we investigate the overhead imposed by the cryptographic operations in our proposed approach.

\subsection{Performance Evaluation}

For the purpose of evaluating ABAKA in a realistic scenario, we created two synthetic datasets based on real world statistics of the population of two cities: New York (USA), focusing on the Manhattan island, and Milan (Italy). In particular, we estimated the average number of ABAKA users in an area of $1 \mathrm{~km}^{2}$, based on: (1) the average population density in such cities, obtained from 35. and $[36$; (2) the statistics on the smartphone penetration in the state of belonging, i.e., the percentage of population owning a smartphone, according to [37] and [38; and (3) a hypothetical percentage of the smartphone users with the ABAKA application installed $(50 \%, 60 \%$, and $70 \%$ were considered). Moreover, in our evaluation we assumed a WiFi range of 25 meters for each device [39]. Table 2 shows some statistics about the considered datasets, in particular the number of users per $\mathrm{km}^{2}$, the percentage of considered collaborating users, and the average number of neighboring collaborators for each user. As we can see form Table 2 the Milan dataset represents a non-dense scenario. Indeed, the average collaborating neighbors per ABAKA user, spans, on average, form 2.99 to only 4.00 , with a percentage of ABAKA users in the smartphone-users population of $57550 \%$ and $70 \%$, respectively. The New York dataset, instead, represents a "best case" scenario, where the average connection degree per ABAKA user is high, e.g., some 23.89 neighbors on average, considering a $60 \%$ ABAKA users in the smartphone-users population. 
Table 2: Statistics on the considered datasets (data extracted from 35 36, 37, 38])

\begin{tabular}{cccccc}
\hline \multirow{2}{*}{ City } & $\begin{array}{c}\text { Inhabitants } \\
\text { per } \mathbf{k m}^{2}\end{array}$ & $\begin{array}{c}\text { Smartphone } \\
\text { Users (\%) }\end{array}$ & $\begin{array}{c}\text { ABAKA } \\
\text { Users (\%) }\end{array}$ & \multicolumn{2}{c}{ Neighboring Users } \\
Average & Std. Dev. \\
\hline \multirow{3}{*}{ New York } & \multirow{2}{*}{27,733} & \multirow{2}{*}{$64 \%$} & 50 & 20.00 & 4.82 \\
& & & 60 & 23.89 & 5.31 \\
& \multirow{2}{*}{$* 3$} & 70 & 27.85 & 5.79 \\
\hline \multirow{2}{*}{ Milan } & \multirow{2}{*}{7,382} & \multirow{2}{*}{$41 \%$} & 50 & 2.99 & 1.99 \\
& & & 60 & 3.37 & 2.08 \\
& & & 70 & 4.00 & 2.24 \\
\hline
\end{tabular}

To evaluate the performance of ABAKA, we measured the average success rate for a query packet to be received by the LBSP, varying the maximum allowed size of the cloaked area, from $100 \mathrm{~m}^{2}$, to $600 \mathrm{~m}^{2}$, with steps increase of $100 \mathrm{~m}^{2}$, as well as the maximum allowed hops number, i.e., 10, 15 and 20 hops.

In our evaluation, we performed our experiments considering two possibilities for a user to forward a message to a neighbor, i.e., she can forward the packet to: (1) the closest neighbor, or (2) a random one. We also considered different possible actions that a user can perform when receiving a packet outside of the largest possible cloaked area. In this case, she can decide to: (i) drop the packet, (ii) forward it to a random neighbor, or (iii) return the packet back to the previous user, which in turn will select another user to which forward the message. However, in our experiments we did not consider option (i), since it would reduce the probability for a message to complete the protocol.

We considered four different types of attributes for the population, reported in Table 3. The table reports also the distribution of attribute values in the population, extracted from [35]. We performed 1000 runs of the ABAKA protocol, each time randomly initializing the configuration according to the values in Table 3 , and randomly selecting a different issuer.

Our evaluation of ABAKA considers the following two different policy combinations, where parentheses delimit a policy enforced on a single message part (considered notation is consistent with the reported attributes in Table 3):

(a) $[(A \geq 18 \wedge S=f),(A \geq 18 \wedge S=f),(A \geq 18 \wedge S=f),(A \geq 18 \wedge S=f)]$ 
Table 3: Considered attributes and their distribution, according to the data in 35

\begin{tabular}{llc}
\hline Attribute & Attribute value & $\begin{array}{c}\text { Presence in the } \\
\text { population (\%) }\end{array}$ \\
\hline \multirow{2}{*}{ Sex $(S)$} & male $(m)$ & 47.5 \\
& female $(f)$ & 52.5 \\
\hline \multirow{3}{*}{ Race $(R)$} & white $(w)$ & 33 \\
& black $(b)$ & 25.5 \\
& latino or hispanic $(h)$ & 28 \\
& asian $(s)$ & 12.7 \\
& american indian $(a)$ & 0.8 \\
\hline \multirow{2}{*}{ Origin $(O)$} & foreign born $(f)$ & 37 \\
& local born $(l)$ & 63 \\
\hline \multirow{3}{*}{ Age $(A)$} & $<18$ & 21.6 \\
& between 18 and 65 & 66.3 \\
& $\geq 65$ & 12.1 \\
\hline
\end{tabular}

(b) $[(A \geq 18 \wedge O=l),(A \geq 18 \wedge R=h)]$

Policies combination (a) provides at least 5-anonymity, and 1-sensitivity, while polcies combination (b) provides at least 3-anonymity and 2-sensitivity.

Figures 4, 5, 6, and 7] present the results of our simulation, adopting the different strategies introduced above, with set of policies (a) on the Milan dataset; Figures 8, 9, 10, and 11 presents the results of our simulation with set of policies (a) on the New York dataset. For the sake of brevity, for policies combination (b) we report only the results obtained on both datasets, with strategy (1) for selecting the next collaborating user, and strategy (iii) to handle the out-of-area case. We report these results in Figure 12 and Figure 13

From our results, we can derive some useful observations. First of all, we notice that, unsurprisingly, the average number of collaborating neighbors per ABAKA user (listed in Table 2 influences the success rate of our proposal. This is more evident if we consider the Milan dataset. As an example, Figure 4 shows ${ }_{615}$ a significative increase of the success rate, i.e., from a maximum of some $60 \%$ to a maximum of some $70 \%$, as the number of ABAKA users (and consequently the number of neighbors per user) grows. However, note that even in non-dense 


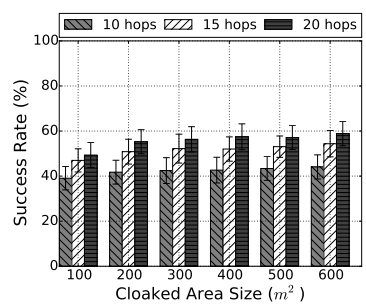

(a) $50 \%$ ABAKA users.

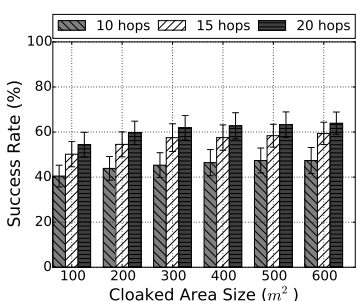

(b) $60 \%$ ABAKA users.

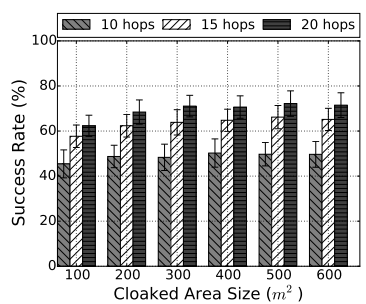

(c) $70 \%$ ABAKA users.

Figure 4: Success rate of ABAKA simulating policies combination (a) on the Milan dataset. Each user forwards the message to its closest neighbor; outside the cloaked area, user returns the message to previous user.

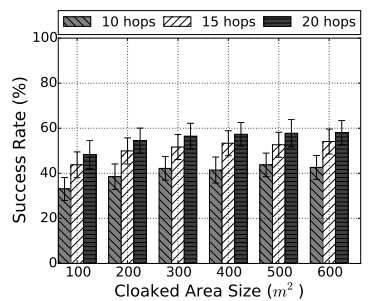

(a) $50 \%$ ABAKA users.

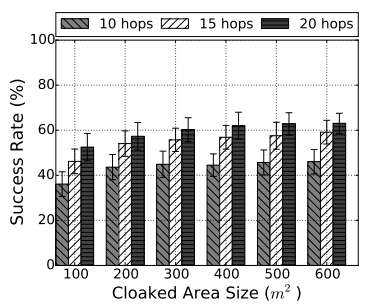

(b) $60 \%$ ABAKA users.

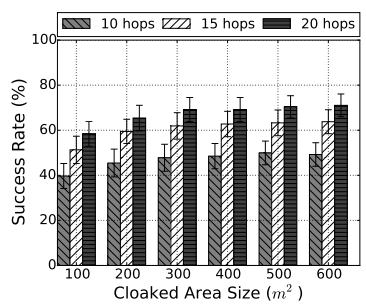

(c) $70 \%$ ABAKA users.

Figure 5: Success rate of ABAKA simulating policies combination (a) on the Milan dataset. Each user forwards the message to its closest neighbor; outside the cloaked area, user forwards the message to a random neighbor.

scenarios, ABAKA achieves a reasonable success rate, e.g., in Figure 4(c) we can observe that ABAKA is capable to achieve a success rate of some $70 \%$, considering a maximum of 20 hops and a maximum cloaked area size of $200 \mathrm{~m}^{2}$.

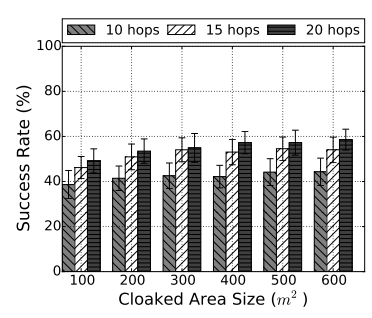

(a) $50 \%$ ABAKA users.

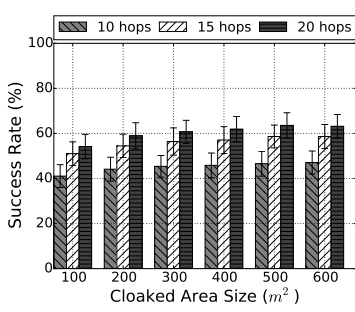

(b) $60 \%$ ABAKA users.

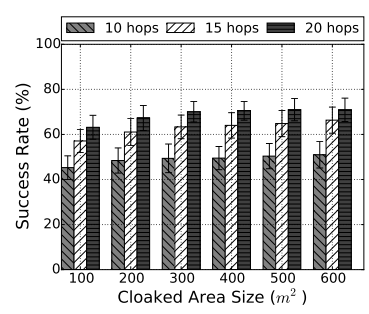

(c) $70 \%$ ABAKA users.

Figure 6: Success rate of ABAKA simulating policies combination (a) on the Milan dataset. Each user forwards the message to a random neighbor; outside the cloaked area, user returns the message to previous user. 


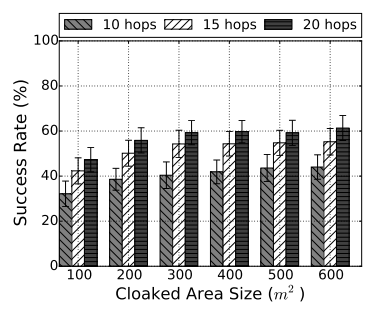

(a) $50 \%$ ABAKA users.

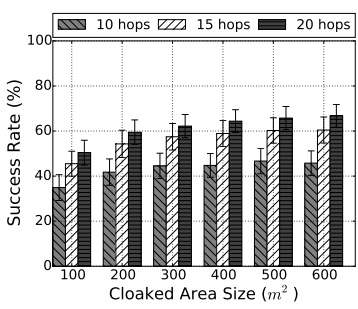

(b) $60 \%$ ABAKA users.

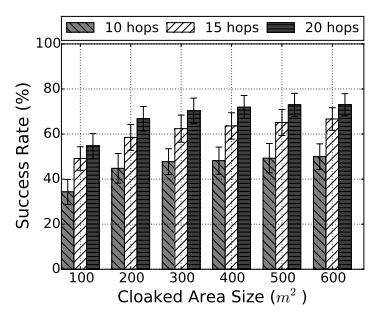

(c) $70 \%$ ABAKA users.

Figure 7: Success rate of ABAKA simulating policies combination (a) on the Milan dataset. Each user forwards the message to a random neighbor; outside the cloaked area, user forwards the message to a random neighbor.

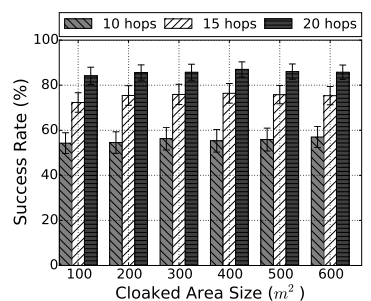

(a) $50 \%$ ABAKA users.

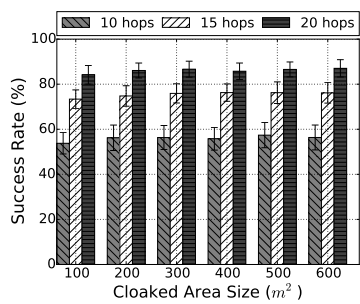

(b) $60 \%$ ABAKA users.

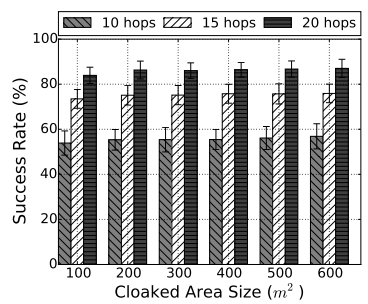

(c) $70 \%$ ABAKA users.

Figure 8: Success rate of ABAKA simulating policies combination (a) on the New York dataset. Each user forwards the message to its closest neighbor; outside the cloaked area, user returns the message to previous user.

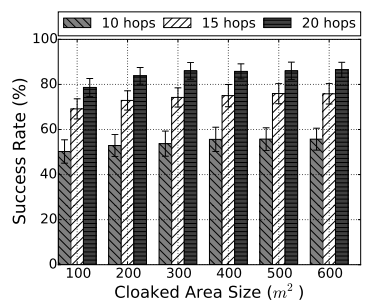

(a) $50 \%$ ABAKA users.

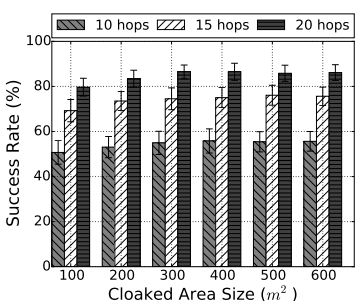

(b) $60 \%$ ABAKA users.

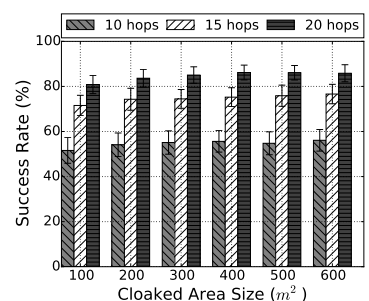

(c) $70 \%$ ABAKA users.

Figure 9: Success rate of ABAKA simulating policies combination (a) on the New York dataset. Each user forwards the message to its closest neighbor; outside the cloaked area, user forwards the message to a random neighbor.

Second, we can observe that both the maximum number of allowed hops, as well as the maximum cloacked area size, play an important role. The effect of the maximum number of hops is evident from the results of the experiment 


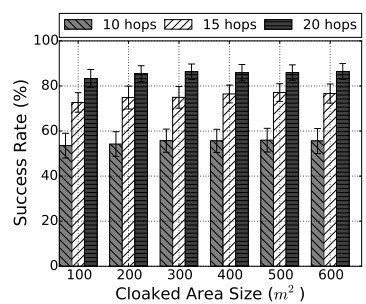

(a) $50 \%$ ABAKA users.

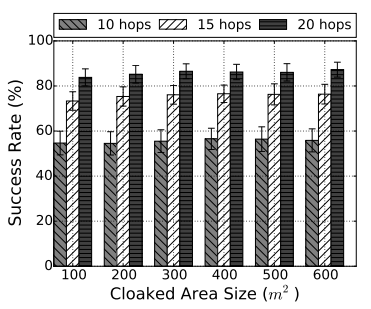

(b) $60 \%$ ABAKA users.

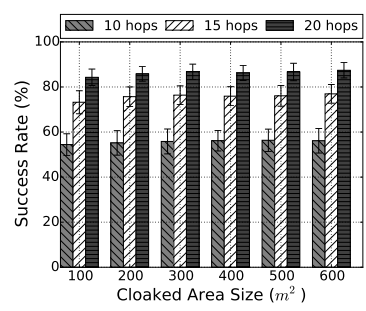

(c) $70 \%$ ABAKA users.

Figure 10: Success rate of ABAKA simulating policies combination (a) on the New York dataset. Each user forwards the message to a random neighbor; outside the cloaked area, user returns the message to previous user.

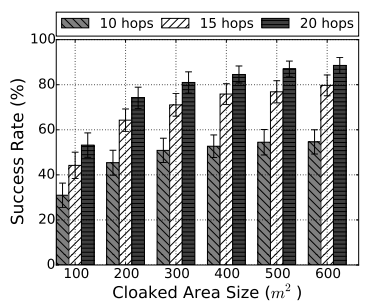

(a) $50 \%$ ABAKA users.

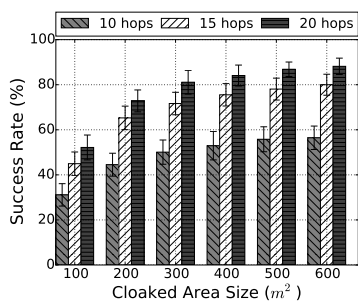

(b) $60 \%$ ABAKA users

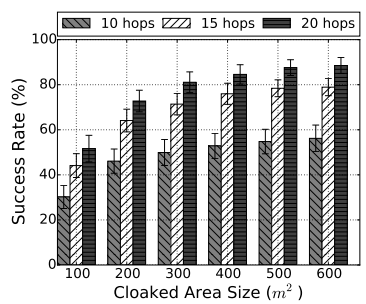

(c) $70 \%$ ABAKA users.

Figure 11: Success rate of ABAKA simulating policies combination (a) on the New York dataset. Each user forwards the message to a random neighbor; outside the cloaked area, user forwards the message to a random neighbor.

performed on the New York dataset. For example, from Figure 11 we can see that adopting a maximum number of hops of 20, brings the success rate of the protocol to greater than $90 \%$, while a maximum of 10 hops leads to a success rate lower than $60 \%$. Analogously, the effect of the adopted bigger maximum cloacked area size can be observed from Figure 4 to Figure 11, as an example, Figure 4(a) shows that, with a maximum of 20 hops, a maximum cloacked area size of $100 \mathrm{~m}^{2}$ leads to an average success rate of some $50 \%$, while when the maximum cloacked area size is $600 \mathrm{~m}^{2}$, the success rate is some $60 \%$ an average.

\subsection{Cryptographic Overhead}

For a thorough evaluation of ABAKA, we estimated the overhead introduced by the cryptographic tools used in our protocol. In particular, we measured

the average time required for encryption and decryption with CP-ABE, RSA, 


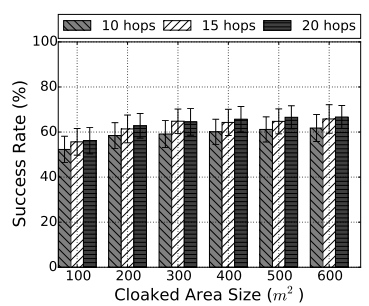

(a) $50 \%$ ABAKA users.

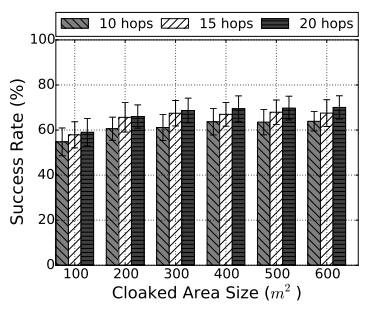

(b) $60 \%$ ABAKA users.

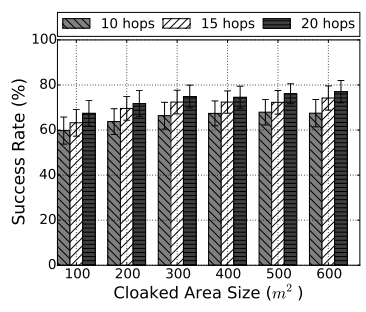

(c) $70 \%$ ABAKA users.

Figure 12: Success rate of ABAKA simulating policies combination (b) on the Milan dataset. Each user forwards the message to its closest neighbor; outside the cloaked area, user returns the message to previous user.

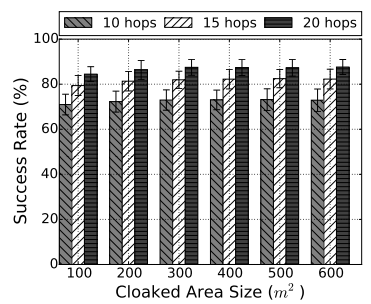

(a) $50 \%$ ABAKA users.

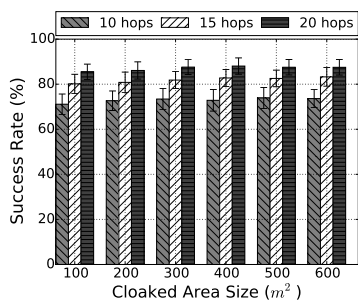

(b) $60 \%$ ABAKA users.

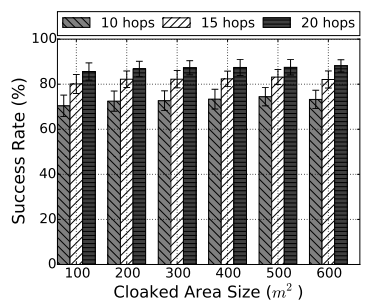

(c) $70 \%$ ABAKA users.

Figure 13: Success rate of ABAKA simulating policies combination (b) on the New York dataset. Each user forwards the message to its closest neighbor; outside the cloaked area, user returns the message to previous user.

and AES-CBC. We considered two different platforms: a laptop equipped with 4x1.8 GHz Intel Core i7-4500U processor, and 8 GB RAM, running Ubuntu 14.04; and a smartphone equipped with a $1.2 \mathrm{GHz}$ dual-core ARM Cortex-A9 CPU processor, and 1 GB RAM, running Android 4.3 "Jelly Bean".

On both platforms, we evaluated $\mathrm{CP}-\mathrm{ABE}$ using the $\mathrm{ABE}$ implementation for Android devices we proposed in 40 [1] Figure 14 shows the results of our measurements on a $250 \mathrm{~KB}$ file (we believe that this is a reasonable size assumption for a piece of query encrypted in the protocol). Since the time required by CP-ABE mainly depends on the number of attributes employed in the cryptographic operations [12, we considered a varying number of attributes for policies and

\footnotetext{
${ }^{1}$ The code of the library is available at http://spritz.math.unipd.it/projects/andraben/
} 
keys from one to 20.

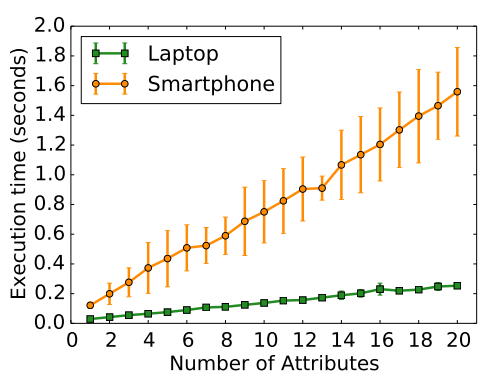

(a) Encryption

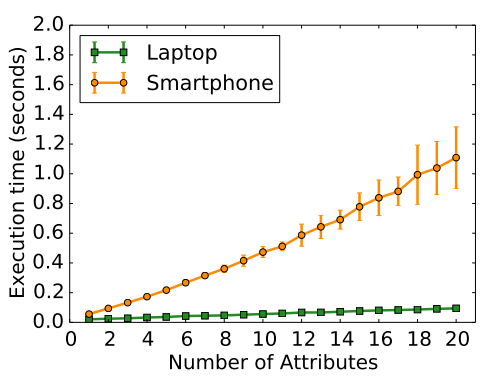

(b) Decryption

Figure 14: Average time required for encryption and decryption operations using CP-ABE on an Android smartphone and a Laptop device.

As we can see from Figure 14, even adopting a large number of attributes, the time required by $\mathrm{CP}-\mathrm{ABE}$ implementation for encryption and decryption is low, on both smartphone and laptop. For a more comprehensive overview of the performance of $\mathrm{ABE}$ on smartphone devices, the reader may refer to our recent work [40]. Additionally, we measured the average encryption and decryption time for RSA, with key size of 4096 bits, and AES-CBC with key size of 256 bits. On both platforms, we employed the openssl library [41], that we cross compiled for Android. We measured RSA encryption and decryption for a key of size 256 bits; while for AES-CBC, we considered a file of size $1 \mathrm{MB}$. Table 4 shows the results of our measurements. As we can see, for both RSA and AES-CBC, the imposed overhead is very small.

Table 4: Average encryption/decryption time for RSA/AES-CBC on Smartphone and Laptop.

\begin{tabular}{ccccc}
\hline \multirow{2}{*}{ Scheme } & \multicolumn{2}{c}{ Smartphone } & \multicolumn{2}{c}{ Laptop } \\
& Encrypt & Decrypt & Encrypt & Decrypt \\
\hline RSA & $7.5101 \mathrm{~ms}$ & $0.0156 \mathrm{~ms}$ & $0.153 \mathrm{~ms}$ & $0.001 \mathrm{~ms}$ \\
AES-CBC $^{*}$ & $26.199 \mathrm{~ms}$ & $26.517 \mathrm{~ms}$ & $2.809 \mathrm{~ms}$ & $3.953 \mathrm{~ms}$ \\
AES-CBC $^{* *}$ & $110.179 \mathrm{~ms}$ & $109.574 \mathrm{~ms}$ & $11.072 \mathrm{~ms}$ & $15.526 \mathrm{~ms}$ \\
\hline
\end{tabular}

* Encryption/decryption of a 250 KByte file

** Encryption/decryption of a 1 MByte file 
The results we obtained confirm the applicability of ABAKA not only on powerful devices such as laptops, but also on smartphone devices. As an example, consider an anonymity level $k=5$, and policies composed by three attributes (which we believe are expressive enough to successfully guarantee $p$-sensitivity). In this case, the average overhead on an Android smartphone would be approximately $(0.27613 \times 5)+0.00751+0.11018=1.49834 \mathrm{~s}$ for the issuer, who has to encrypt the query with a symmetric key, that in turn is encrypted with LBSP's public key (this is a common usage of public key encryption), and encrypt each part of the split message with CP-ABE. Each collaborating user has to decrypt a part of the query with her CP-ABE private key, and immediately encrypt it with AES-CBS. Therefore, the approximate overhead will be $0.13275+0.26199=0.15894 \mathrm{~s}$. Finally, the last collaborating user have to decrypt all the parts that are previously encrypted with AES-CBC. Therefore, she will incur in an additional overhead of $0.02651 \times 5=0.13255 \mathrm{~s}$.

\section{Related Work}

The concept of $k$-anonymity was first introduced for databases applications [42, and later applied in the context of LBSs [5]: the user's position is translated into a cloaked area and provided to the LBSP along with the requested query. The concept of $k$-anonymity has been extended in several aspects, e.g., l-diversity [43, and t-closeness [4]. Moreover, in [9] the authors proposed a $p$-sensitive approach for LBSs, which provides query $l$-diversity by classifying queries into sensitive and non-sensitive groups. However, unlike our work, none of these approaches considered both (i) query semantics, and (ii) sensitive profile attributes of each user, at the same time.

Bamba et al. 45] proposed an approach to provide $k$-anonymity and location $l$-diversity for LBS users. In this scheme, mobile users are not identifiable from $k-1$ other users in a set of $l$ different physical locations such as hospitals, bars and university. This scheme utilizes one or more anonymization servers between users and LBSP to perform spatio-temporal cloaking. 
In traditional approaches for $k$-anonymity in LBSs, the computation of the cloaked area is carried out by an anonymization server to which the query is first forwarded. Such solutions are typically referred as TTP-based schemes. However, the use of a centralized anonymizer offers a single point of attack, and may represent a serious bottleneck for the overall system. To overcome these limitations, researchers proposed several distributed solutions that compute the cloaked area in a collaborative way, referred to as TTP-free solutions. For an overview of the main existing TTP-free solutions, the reader can refer to [46].

Unfortunately, most of the existing schemes (both TTP-free and TTP-based) do not consider the background knowledge of the attackers, except from only a few recently proposed approaches [11. However, an attacker with background information about a user's profile might be able to identify her, even if her location is hidden [47. $k$-anonymity preserving solutions try to overcome the above issues, by considering user profiles information [6, 48. However, unlike our work, all the aforementioned profile-based schemes are centralized, and might be subject to the limitations introduced before. To the best of our knowledge, our proposal is the first TTP-free approach for $p$-sensitive profile $k$-anonymity in LBS that considers user's profile attributes.

\section{Conclusions}

Location and identity privacy in Location-Based Services are major concerns for users who want to protect their privacy from a malicious LBSP, as well as from an eavesdropper. While several solutions for guaranteeing privacy in LBSs have been proposed in the literature, they are often centralized, or do not take into account the prior knowledge of the attacker about user profiles. In this paper we present ABAKA, our collaborative solution that guarantees $k$-anonymity, as well as $p$-sensitivity in LBSs, taking into account the issued query semantics. In our approach, users have a set of attributes associated to their profile. Their attributes are bound to a CP-ABE private key. An LBS 
route to the LBSP. ABAKA enables each issuer to delimit a cloaked area within which she wants to be anonymous, and to specify a list of $k-1$ policies, i.e., attribute combinations, that users in the multi-hop path must satisfy in order to forward the query message to the LBSP. ABAKA provides the possibility of performing a trade-off between the stringency of privacy protection and quality of service for the issuer in her current location, based on the query semantics. We addressed the threat of active and passive adversaries by means of CP-ABE and multi-hop routing approaches. We simulated our protocol on synthetic datasets derived from real population statistics (considering two cities: New York (USA), and Milan (Italy)), and demonstrated that our approach is feasible and efficient.

\section{Acknowledgments}

Mauro Conti is supported by a Marie Curie Fellowship funded by the European Commission (agreement PCIG11-GA-2012-321980). This work is also partially supported by the EU TagItSmart! Project (agreement H2020-ICT302015-688061), the EU-India REACH Project (agreement ICI+/2014/342-896), the Italian MIUR-PRIN TENACE Project (agreement 20103P34XC), and by the projects "Tackling Mobile Malware with Innovative Machine Learning Techniques", "Physical-Layer Security for Wireless Communication", and "Content Centric Networking: Security and Privacy Issues" funded by the University of Padua.

\section{References}

[1] H. A. Karimi, Advanced Location-based Technologies and Services, CRC Press, 2013.

[2] M. Wernke, P. Skvortsov, F. Dürr, K. Rothermel, A classification of location privacy attacks and approaches, Personal and Ubiquitous Computing 18 (1) (2014) 163-175. 
[3] M. Conti, J. Willemsen, B. Crispo, Providing source location privacy in wireless sensor networks: A survey, IEEE Communications Surveys and Tutorials 15 (3) (2013) 1238-1280.

[10] A. Solanas, F. Sebé, J. Domingo-Ferrer, Micro-aggregation-based heuristics for p-sensitive k-anonymity: one step beyond, in: Proceedings of the 2008 International Workshop on Privacy and Anonymity in Information Society, PAIS'08, 2008, pp. 61-69.

[11] R. Shokri, G. Theodorakopoulos, P. Papadimitratos, E. Kazemi, J.-P. Hubaux, Hiding in the mobile crowd: Location privacy through collab- 
oration, IEEE Transactions on Dependable and Secure Computing 11 (3) (2014) 266-279.

[12] J. Bethencourt, A. Sahai, B. Waters, Ciphertext-policy attribute-based encryption, in: Proceedings of the IEEE Symposium on Security and Privacy, S\&P'07, 2007, pp. 321-334.

[13] A. Sahai, B. Waters, Fuzzy identity-based encryption, in: Advances in Cryptology EUROCRYPT 2005, Vol. 3494 of Lecture Notes in Computer Science, 2005, pp. 457-473.

[14] T. Dargahi, M. Ambrosin, M. Conti, N. Asokan, Abaka: A novel attributebased k-anonymous collaborative solution for lbss, Computer Communications 85 (2016) 1-13.

[15] T. Yang, C. Tang, L. Yu, W. Xin, Y. Deng, J. Hu, Z. Chen, VLSP: enabling location privacy in vehicular location based services, in: Proceedings of the 2011 First International Conference on Instrumentation, Measurement, Computer, Communication and Control, MCCC'11, 2011, pp. 462-465.

[16] C. Boldrini, M. Conti, F. Delmastro, A. Passarella, Context-and social-aware middleware for opportunistic networks, Journal of Network and Computer Applications 33 (5) (2010) 525-541.

[17] H. Nishiyama, M. Ito, N. Kato, Relay-by-smartphone: realizing multihop device-to-device communications, IEEE Communications Magazine 52 (4) (2014) 56-65.

[18] M. Conti, F. Delmastro, G. Minutiello, R. Paris, Experimenting opportunistic networks with wifi direct, in: Wireless Days, WD'13, IEEE, 2013, pp. $1-6$.

[19] E. Biondi, C. Boldrini, A. Passarella, M. Conti, Optimal duty cycling in mobile opportunistic networks with end-to-end delay guarantees, in: Proceedings of the 20th European Wireless Conference, European Wireless'14, VDE, 2014, pp. 1-6. 
[20] C. A. Ardagna, M. Conti, M. Leone, J. Stefa, An anonymous end-to-end communication protocol for mobile cloud environments, IEEE Transactions on Services Computing 7 (3) (2014) 373-386.

[21] X. Bao, Y. Lin, U. Lee, I. Rimac, R. R. Choudhury, Dataspotting: Exploiting naturally clustered mobile devices to offload cellular traffic, in: Proceedings of the IEEE International Conference on Computer Communications, INFOCOM'13, IEEE, 2013, pp. 420-424.

[22] M. Conti, L. Zhang, S. Roy, R. Di Pietro, S. Jajodia, L. V. Mancini, Privacypreserving robust data aggregation in wireless sensor networks, Security and Communication Networks 2 (2) (2009) 195-213.

[23] H. Gao, C. H. Liu, W. Wang, J. Zhao, Z. Song, X. Su, J. Crowcroft, K. K. Leung, A survey of incentive mechanisms for participatory sensing, Communications Surveys \& Tutorials, IEEE 17 (2) (2015) 918-943.

[24] Q. Li, G. Cao, Providing privacy-aware incentives for mobile sensing, in: Proceedings of the International Conference on Pervasive Computing and Communications, PerCom'13, IEEE, 2013, pp. 76-84.

[25] M. Conti, C. Boldrini, S. S. Kanhere, E. Mingozzi, E. Pagani, P. M. Ruiz, M. Younis, From manet to people-centric networking: Milestones and open research challenges, Computer Communications 71 (2015) 1-21.

[26] J. Li, Q. Huang, X. Chen, S. S. M. Chow, D. S. Wong, D. Xie, Multiauthority ciphertext-policy attribute-based encryption with accountability, in: Proceedings of the 6th ACM Symposium on Information, Computer and Communications Security, ASIACCS'11, 2011, pp. 386-390.

[27] R. Shokri, J. Freudiger, J.-P. Hubaux, A unified framework for location privacy, Tech. rep. (2010).

[28] C. A. Ardagna, A. Stavrou, S. Jajodia, P. Samarati, R. Martin, A multi-path approach for k-anonymity in mobile hybrid networks, in: Proceedings of 
the International Workshop on Privacy in Location-Based Applications, PILBA'08, 2008, pp. 82-101.

[29] H. Takabi, J. B. Joshi, H. Karimi, et al., A collaborative k-anonymity approach for location privacy in location-based services, in: Proceedings of the 5th International Conference on Collaborative Computing: Networking, Applications and Worksharing, CollaborateCom'09, 2009, pp. 1-9.

[30] X. Gong, X. Chen, K. Xing, D.-H. Shin, M. Zhang, J. Zhang, Personalized location privacy in mobile networks: A social group utility approach, INFOCOM'15, IEEE, 2015, pp. 1008-1016.

[31] G. Zhong, U. Hengartner, Toward a distributed k-anonymity protocol for location privacy, in: Proceedings of the 9th Annual ACM Workshop on Privacy in the Electronic Society, WPES'08, 2008, pp. 33-38.

[32] G. Zhong, U. Hengartner, A distributed k-anonymity protocol for location privacy, in: IEEE International Conference on Pervasive Computing and Communications, PerCom'09, 2009, pp. 1-10.

${ }_{840}$ [33] M. G. Reed, P. F. Syverson, D. M. Goldschlag, Anonymous connections and onion routing, IEEE Journal on Selected Areas in Communications 16 (4) (1998) 482-494.

[34] D. Rebollo-Monedero, J. Forné, A. Solanas, A. Martínez-Ballesté, Private location-based information retrieval through user collaboration, Computer Communications 33 (6) (2010) 762-774.

[35] United states census bureau - quick facts, http://quickfacts.census gov/qfd/states/36/36061.html.

[36] Focus on milan, 2012, http://allegati.comune.milano.it/Statistica/ AnnuariStatistici/MilanoInBreve2012/FocusOnMilano2012.pdf.

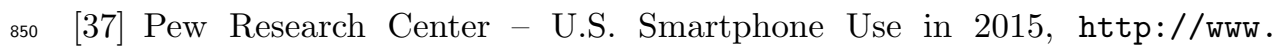
pewinternet.org/2015/04/01/us-smartphone-use-in-2015/. 
[38] Lo scenario social, digital e mobile in europa e in italia,

n http://www.wired.it/internet/social-network/2014/02/17/ lo-scenario-social-digital-e-mobile-europa-e-italia/

[46] A. Khoshgozaran, C. Shahabi, A taxonomy of approaches to preserve location privacy in location-based services, International Journal of Computational Science and Engineering 5 (2) (2010) 86-96. 
[47] R. Shokri, C. Troncoso, C. Diaz, J. Freudiger, J.-P. Hubaux, Unraveling an old cloak: K-anonymity for location privacy, in: Proceedings of the 9th Annual ACM Workshop on Privacy in the Electronic Society, WPES'10, 2010, pp. 115-118.

[48] X. Chen, J. Pang, Protecting query privacy in location-based services, GeoInformatica 18 (1) (2014) 95-133. 